\title{
Development of the MFPR model for fission gas release in irradiated $\mathrm{UO}_{2}$ under transient conditions
}

\author{
Michael S. Veshchunov and Vladimir I. Tarasov* \\ Nuclear Safety Institute (IBRAE), Russian Academy of Sciences, 52, B. Tulskaya, 115191 Moscow, Russia
}

Received: 3 October 2015 / Received in final form: 29 June 2016 / Accepted: 6 December 2016

\begin{abstract}
The fission gas release microscopic model of the mechanistic code MFPR is further developed for modelling of enhanced release from irradiated $\mathrm{UO}_{2}$ fuel under transient conditions of the power ramp tests, along with the microstructure evolution characterised by the formation of a new population of large intragranular bubbles with a rather wide size distribution (from 30 to $500 \mathrm{~nm}$ ), observed in transient-tested $\mathrm{UO}_{2}$ fuel samples. Implementation of the additional microscopic mechanisms results in a notable improvement of the code predictions (in comparison with the previous code version) for the fractional gas release in the Ris $\varnothing$ ramp tests with three different hold times of 3,40 and $62 \mathrm{~h}$ at the terminal linear power of $\approx 40 \mathrm{~kW} / \mathrm{m}$.
\end{abstract}

\section{Introduction}

For realistic description of fission-gas release and fuel swelling as a function of fuel-fabrication variables and in a wide range of reactor operating conditions, mechanistic models must treat them as coupled phenomena and must include various microscopic mechanisms influencing fission gas behaviour. The key point in such analysis is intragranular bubbles behaviour, which is strongly influenced by irradiation and/or thermal treatment conditions.

A relatively simple behaviour under steady irradiation conditions, characterised by a population of small nanometre bubbles with a relatively narrow size distribution function, becomes much more complicated under transient conditions (power ramps). For consistent modelling of bubbles behaviour under transient conditions, the critical assessment of available models, their modification and development of more advanced models for implementation in the mechanistic codes, become rather important task. In particular, the improved model for the irradiation induced resolution of gas atoms from bubbles, which allows a reasonable interpretation of a broad "trimodal" bubble size distribution (including gas atoms and two populations of bubbles), observed in the transient-tested fuel pellets [1], and the additional gas transport mechanisms necessary for correct description of the enhanced gas release in these tests, were recently developed by the authors [2].

After implementation of the new models in the mechanistic code MFPR, which was developed in collaboration between IBRAE and IRSN (Cadarache, France)

\footnotetext{
* e-mail: tarasov@ibrae.ac.ru
}

$[3,4]$ and is currently the constituent part of the advanced fuel performance and safety code SFPR [5], the refined code MFPR was applied in [2] to the self-consistent consideration of the fuel microstructure evolution and fission gas release under conditions of ramp tests $[1,6]$. Despite a significant improvement of the code predictions after implementation of the set of microscopic mechanisms effectively operating under transient conditions, there were still some deficiencies in the description of fission gas release from the innermost central zone of the fuel pellet ( $x=r / R_{\text {pellet }}<0.1$, where $R_{\text {pellet }}$ is the pellet radius), which required engaging of additional mechanisms.

In the current paper the main microscopic mechanisms of the intragranular bubble system evolution and fission gas release considered in [2] are briefly overviewed. Besides, additional mechanisms of bubbles transport to grain boundaries, considered earlier in MFPR in application to post-irradiation annealing regimes [7], were adapted to transient conditions and applied to analysis of the fission gas release in the tests [6].

\section{Microstructure evolution}

Transmission electron microscopy (TEM) has been used in an extensive study [1] of the microstructure of baseirradiated and transient-tested samples of LWR nuclear fuels. The steady state base irradiation of $3 \%$ enriched $\mathrm{UO}_{2}$ fuel was performed at a maximum linear power of $26 \mathrm{~kW} / \mathrm{m}$ (corresponding to the fission rate $\approx 1.3 \times 10^{13}$ fissions $/ \mathrm{s} \mathrm{cm}^{3}$ ) to an average burn-up of $4.5 \%$ FIMA. The transient-tested samples came from pellets of the baseirradiated fuel which had been further subjected in reactor 
to power increases up to a maximum of $42 \mathrm{~kW} / \mathrm{m}$ (fission rate $\approx 2.1 \times 10^{13}$ fissions $/ \mathrm{s} \mathrm{cm}^{3}$ ) with the hold time up to $62 \mathrm{~h}\left(=2.2 \times 10^{5} \mathrm{~s}\right)$.

Under the steady-state irradiation conditions most of the fission gas produced was retained in solution in the fuel matrix or precipitated into small fission bubbles with a narrow size distribution and an average diameter of $8 \mathrm{~nm}$. The bubble spatial distribution was homogeneous, with an average concentration of $(1.2-1.9) \times 10^{22} \mathrm{~m}^{-3}$.

The effect of the transient test was to increase the fuel centre temperature from $0.45 T_{\mathrm{m}} \approx 1290^{\circ} \mathrm{C}$ to about $0.56 T_{\mathrm{m}} \approx 1600^{\circ} \mathrm{C}$, causing significant changes to fuel microstructure. The major microstructure change in the fuel centre resulting from the transient was the formation of a new population of large fission bubbles with a broad bubble size distribution (30-500 $\mathrm{nm}$ in diameter) and an average bubble concentration of $7 \times 10^{18} \mathrm{~m}^{-3}$. The temperature rise at the fuel periphery, on the other hand, was small and the microstructure remained essentially similar to that of the base-irradiated fuel, with similar density and distribution of small fission bubbles.

Since the temperature rise was high in the fuel centre and small at the periphery, a relatively large temperature gradient was attained in the fuel pellet. This activates the biased migration of bubbles and may additionally accelerate their coalescence, especially in the high-temperature central zone where bubbles mobility is high, resulting in formation of the large-size bubble population.

Three mechanisms are considered in MFPR that could lead to the bubble biased migration in the fuel is as follows [8]: (1) evaporation-condensation across the bubble, (2) bubble surface diffusion, and (3) mass diffusion around the bubbles; these mechanisms are tightly connected with the corresponding mechanisms of the bubble random migration. If the bubbles are small, the surface diffusion process would be expected to dominate at lower temperatures. For relatively large bubbles and at high temperatures the evaporation-condensation process is expected to be the dominant process.

For proper consideration of the bubble population with wide size distribution function (SDF) a multimodal option of the MFPR code [3,4], rather than the base bimodal option, was used in further calculations. In this approach the problem is formulated in terms of Smoluchowski equation with the coagulation kernel describing the rate of interaction between particles $i$ and $j$ in the form:

$$
K_{i j}=4 \pi\left(D_{i}+D_{j}\right)\left(R_{i}+R_{j}\right)+\pi\left(R_{i}+R_{j}\right)^{2}\left|v_{i}-v_{j}\right|,
$$

where first and the second terms in the rhs relate to the random and biased bubble migration mechanisms. In this equation, $D_{i}$ and $R_{i}$ are respectively the bubble diffusivity and radius, whereas $v_{i}$ is the bubble velocity, which is proportional to the temperature gradient.

Besides, the modification of Nelson's model $[9,10]$ for irradiation induced gas atom resolution from bubbles proposed by the authors in [11] (and re-considered in [2]) was implemented in the code. In this modified model, a tendency of gas atoms ejected from a bubble into the matrix to return back to the bubble by diffusion within the re-solution layer $\delta$ (of several $\mathrm{nm}$ ) was taken into quantitative consideration. As a result, the rate equation for the variation of the number of gas atoms $N_{b}$ in the bubble of radius $R_{b}$ takes form

$$
\begin{aligned}
\frac{d N_{b}}{d t} & =4 \pi D_{g} c_{g} R_{b}-\omega_{\text {res }} N_{b} \frac{\delta}{R_{b}+\delta} \\
& =4 \pi D_{g} c_{g} R_{b}-\omega^{\prime}{ }_{r e s} N_{b},
\end{aligned}
$$

where $c_{g}$ and $D_{g}$ are the concentration and diffusivity of gas atoms in the matrix, respectively,

$$
\omega^{\prime}{ }_{r e s} \approx \omega_{\text {res }} \frac{\delta}{R_{b}+\delta} \approx G b_{0}\left(\frac{3 \lambda}{3 \lambda+R_{b}}\right)\left(\frac{\delta}{R_{b}+\delta}\right)=b^{\prime} G,
$$

thus leading to an additional factor $\delta /\left(R_{b}+\delta\right)$ for the resolution rate $\omega_{\text {res }}=b G$, which was derived by Nelson disregarding the back influx of the ejected atoms, $G$ is the fission rate, $b_{0} \approx(2-3) \times 10^{-23} \mathrm{~m}^{3}$ was evaluated from the tests of Cornell and Turnbull [12,13], $\lambda \approx 1-1.5 \mathrm{~nm}$.

In order to elucidate the effect of the new resolution model, calculations using the MFPR versions with the original Nelson model and the modified one were run. For the steady state irradiation period of the test the code qualitatively correctly reproduced the bimodal bubble size distribution, i.e. gas atoms and a population of small bubble with a narrow SDF.

Results of the calculations for the transient conditions with the original and modified Nelson models are compared in Figure 1 for two relative distances $x=r / R_{\text {pellet }}$ from the pellet centre. In the pellet low temperature periphery $(x=1)$ the both models predict narrow SDFs located near $2 \mathrm{~nm}$, which are close to each other. In this location the SDFs turned out to be close to those calculated for the baseirradiated fuel.

The maximum bubble coalescence effect and the broadest SDF was found at a distance of $x \sim 0.2$ from the pellet centre where some decrease of the temperature (by a few tens of $\mathrm{K}$ ) in comparison with that in the centreline is compensated with an excess induced by the biased migration in the temperature gradient (which is zero at the pellet centreline). In this case the SDFs for the two resolution models differ qualitatively: while with the original model the SDF has mainly the bimodal shape with the bubble sizes mainly in the range $10-20 \mathrm{~nm}$, the modified model distinctly predicts formation of two separate bubble populations. The population of smaller bubbles is characterized by the mean bubble size of $3.1 \mathrm{~nm}$ and the total density of $\sim 1.1 \times 10^{20} \mathrm{~m}^{-3}$. The mean bubble size of the second population was found to be of $131 \mathrm{~nm}$ whereas the total density turned out to be of $\sim 2.4 \times 10^{19} \mathrm{~m}^{-3}$ which is in reasonable agreement with the experimental value of $\sim 0.7 \times 10^{19} \mathrm{~m}^{-3}$, taking into account uncertainties in the model parameters and irradiation conditions.

Besides, MFPR predicts significant fractional fission gas release (up to $\approx 70 \%$ in the pellet hot zone); however, it was not controlled in this test. For this reason, gas release measurements in the Ris $\varnothing$ Project tests [6] performed under similar transient conditions will be analysed in the next section. 


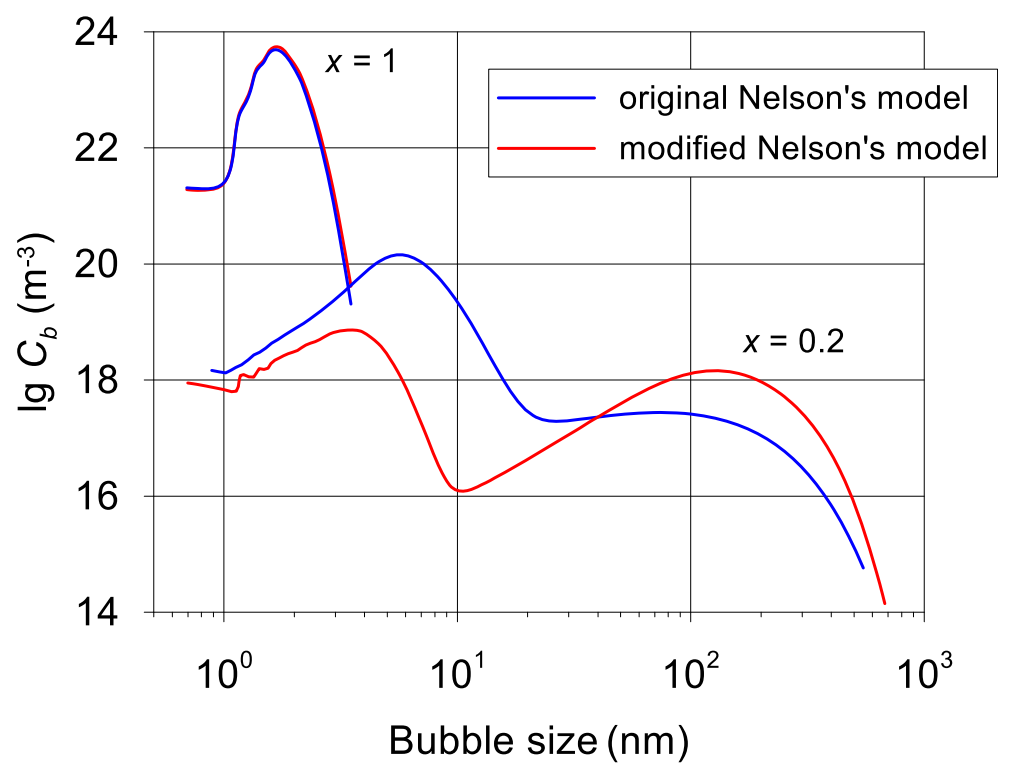

Fig. 1. Bubble SDF calculated with two variants of the resolution model under conditions of the transient test [1].

\section{Fission gas release}

Transient tests $[6]$ have been carried out in Ris $\varnothing$ Project to explore fuel performance at increasing burn-up levels to $\sim 45 \mathrm{GWd} / \mathrm{t}(\sim 4.7 \%$ FIMA $)$ and beyond, especially for power increases (transients) late in life. The tests were performed with the PWR design fuel pellet of diameter $9 \mathrm{~mm}$, pellet density $93.7 \%$ TD and grain diameter $6 \mu \mathrm{m}$. The fuel had been irradiated in the Biblis-A reactor (Germany) to burn-up of 4.3-4.4\% FIMA (pin average). The highest linear power seen by this fuel was $26.7 \mathrm{~kW} / \mathrm{m}$. The fuels did not release more than $0.3 \%$ of their fission gas inventory during the base irradiation. Transient test was carried out in the DR3 reactor at Ris $\emptyset$. The approach to the terminal power was made either in two large jumps or in multiple steps of 2 or $5 \mathrm{~kW} / \mathrm{m}$.

In all of the instrumented tests, the fuel centre temperature and the fission gas pressure in the plenum were simultaneously measured. The temperature of the cladding surface was $613 \mathrm{~K}$ in case of PWR fuel and $563 \mathrm{~K}$ in the case of the BWR fuel, whereas the conservative upper limits of fuel centreline temperature were estimated in $[6]$ to range from $\sim 1773 \mathrm{~K}$ at linear heat rating $30 \mathrm{~kW} / \mathrm{m}$ to $\sim 2143 \mathrm{~K}$ at linear heat rating $40 \mathrm{~kW} / \mathrm{m}$. Post-test examinations of the specimens allowed plotting fractional gas releases and grain growth as functions of terminal local fuel temperature, which was calculated in [6].

The modified MFPR code version was applied to calculation of the release curves for three representative sections of transient-tested fuel with the hold times 4, 40 and $62 \mathrm{~h}$ at linear heat rating $(\mathrm{LHR}) \approx 40 \mathrm{~kW} / \mathrm{m}$. The temperature radial profiles in fuel pellets were considered as parabolic with the centreline temperature of $T_{c} \approx 2100 \mathrm{~K}$ and the pellet outer surface temperature of $T_{\text {swf }} \approx 1100 \mathrm{~K}$, in accordance with temperature evaluation in [6]. High temperatures attained in the fuel centreline induced very steep radial thermal gradient (up to $\sim 4 \times 10^{3} \mathrm{~K} / \mathrm{cm}$ ), which made the bubble-biased migration in the temperature gradient an important mechanism of gas release.
In order to elucidate inputs of various release mechanisms in the transient tests [6], calculations for the hold time of $62 \mathrm{~h}$ with different options of MFPR were carried out (Fig. 2).

The calculation run with various bubble transport mechanisms switched off characterizes the gas release solely by the gas atom diffusion mechanism, which occurs to be relatively weak $\approx 35 \%$ (at $x=0$ ), curve 1 .

Switching on of the sweeping mechanism by grain boundaries results in the significant increase of the gas release to $\approx 63 \%$, curve 2 , demonstrating the important contribution of this mechanism to the total gas release under transient conditions. An adequacy of the MFPR advanced grain growth model $[14,15]$ used in these calculations was validated by comparison of the local grain sizes measured at different radial positions of the tested fuel pellet [6] to calculations (Fig. 3).

Random migration of bubbles (by thermal diffusion) gives a relatively weak input to the gas release (a few percent) in the current calculations (at variance with [2] where the gas release during the base irradiation was notably overestimated and thus the gas content in the fuel correspondingly underestimated), which for this reason is not presented in Figure 2. In contrast, consideration of the bubble-biased migration in the temperature gradient significantly enhances the calculated gas release, curve 3 . However, in the innermost zone of the cylindrical pellet, $x<0.1$, where the thermal gradient is small, the bubble biased migration decreases and tends to zero in the pellet centre, significantly reducing the gas release in this zone, in an apparent contradiction with observations.

In order to overcome this shortcoming, the model is further improved (in comparison with the previous version [2]). Namely, additional mechanisms of bubbles transport to grain boundaries considered earlier in MFPR in application to post-irradiation annealing regimes [7] and associated with the bubble biased migration in the point defect concentration gradient and entrainment by moving dislocations, are adapted to transients. 


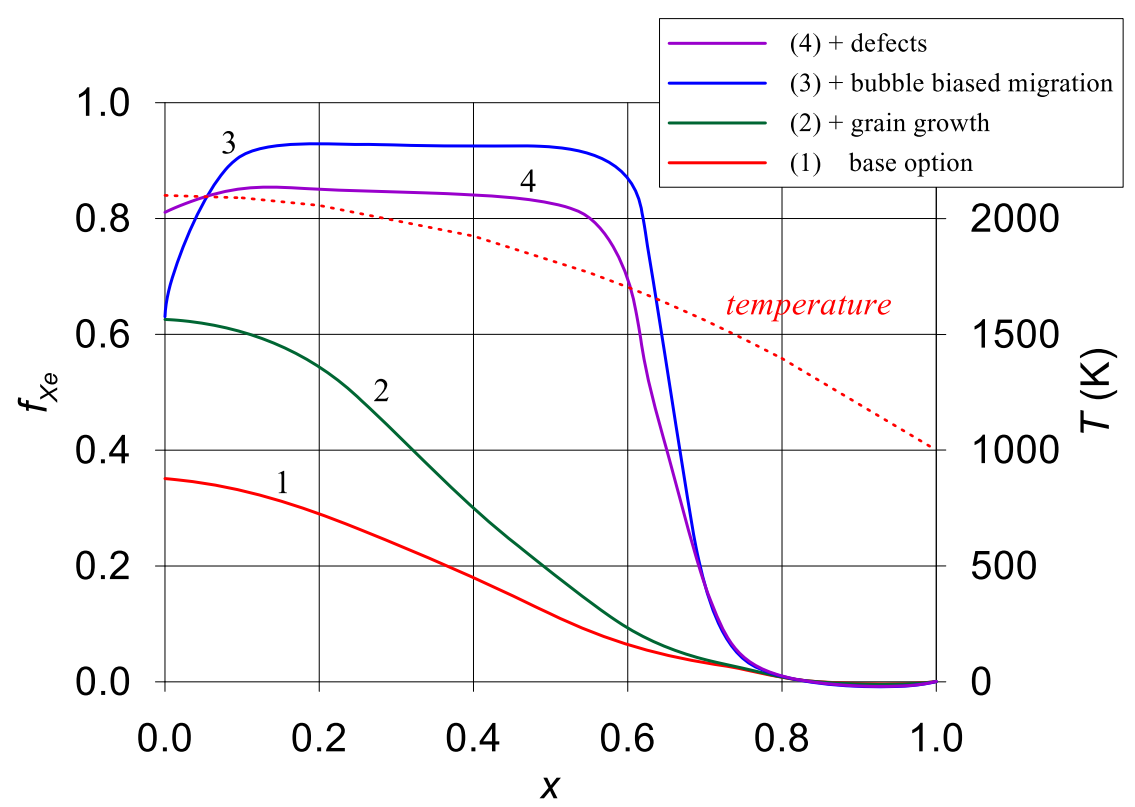

Fig. 2. The radial distribution of fractional gas release for the hold time of $62 \mathrm{~h}$, calculated with different options of the code.

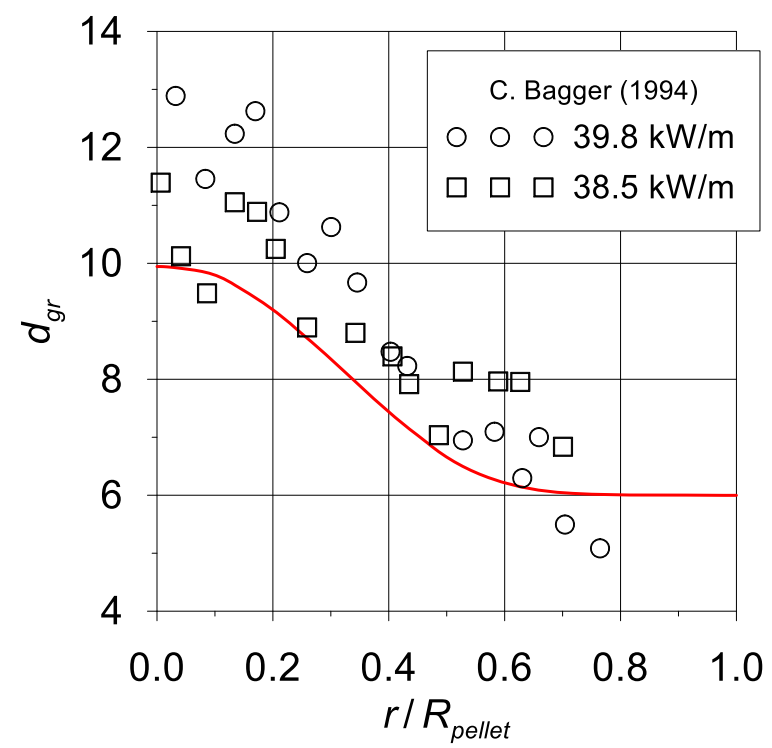

Fig. 3. The radial distribution of $\mathrm{UO}_{2}$ grain size measured in [6] (markers) and calculated by MFPR (curve).

For this purpose one should replace the simplified steady state rate equations for point defects with more realistic diffusion equations. In this case the evolution of the vacancy distribution in a spherical grain of radius $R_{g r}$ under irradiation conditions is described in terms of the bulk concentration, $c_{v}$ (number of vacancies per unit volume), by diffusion equation

$$
\frac{\partial c_{v}}{\partial t}=D_{v} \Delta c_{v}-k_{v}^{2} D_{v} c_{v}-\alpha D_{i} c_{i} c_{v}+K\left(1-\zeta_{l}\right)+K_{e}+K_{p},
$$

where the sink terms in the r.h.s. were described in detail e.g. in $[3,4]$, and by a similar diffusion equation for the bulk concentration of interstitials, $c_{i}$.
As shown in [16], at high temperatures $>1500^{\circ} \mathrm{C}$ the thermal effects for vacancies and interstitials dominate over the radiation ones, $K_{e} \gg K$, resulting in $c_{v} \approx c_{v}^{(e q)}$ and $D_{v} c_{v} \approx D_{v} c_{v}^{(e q)} \gg D_{i} c_{i}$. In this case, the system of point defects becomes "quasi-equilibrium" and behaves similarly to that under high-temperature annealing conditions (i.e. without irradiation). Owing to the enhanced coalescence of bubbles during transient, observed in the tests [1] and predicted by calculations (above), and taking into account that the total volume of bubbles increases owing to coalescence under "quasi-equilibrium" conditions, this requires persistent supply of vacancies from extended defects (grain boundaries and dislocations).

In the initial stage of the bubbles volume growth, when dislocations are the main source of vacancies, evaporation of vacancies (necessary for growing bubbles equilibration) from a dislocation enhances its non-conservative motion (climb), which, in its turn, results in capturing (sweeping) of intragranular bubbles by the moving dislocation. This explains the dislocation creep observed under transient conditions [1], on the one hand, and offers the additional transport mechanism of bubbles to grain boundaries by climbing dislocations (similarly to the annealing tests), on the other hand.

After some time a strong pinning of dislocations by swept bubbles can saturate this source of point defects [7], and grain boundaries become the dominant source of vacancies during the subsequent period of the transient tests. In this situation a vacancy flux directed from the grain boundaries to the grain interior arises and induces bubble biased migration up the vacancy concentration gradient with the velocity $v_{b}^{(v a c)}(r)=-2 D_{v} \partial c_{v} / \partial r$ (the so called Evans mechanism), resulting in the additional gas release during the late stage of transient. However, in the present calculations this effect is rather weak and the main contribution (notably increasing fission gas release in the innermost central zone of the pellet) is afforded by the dislocation creep mechanism, curve 4 . 


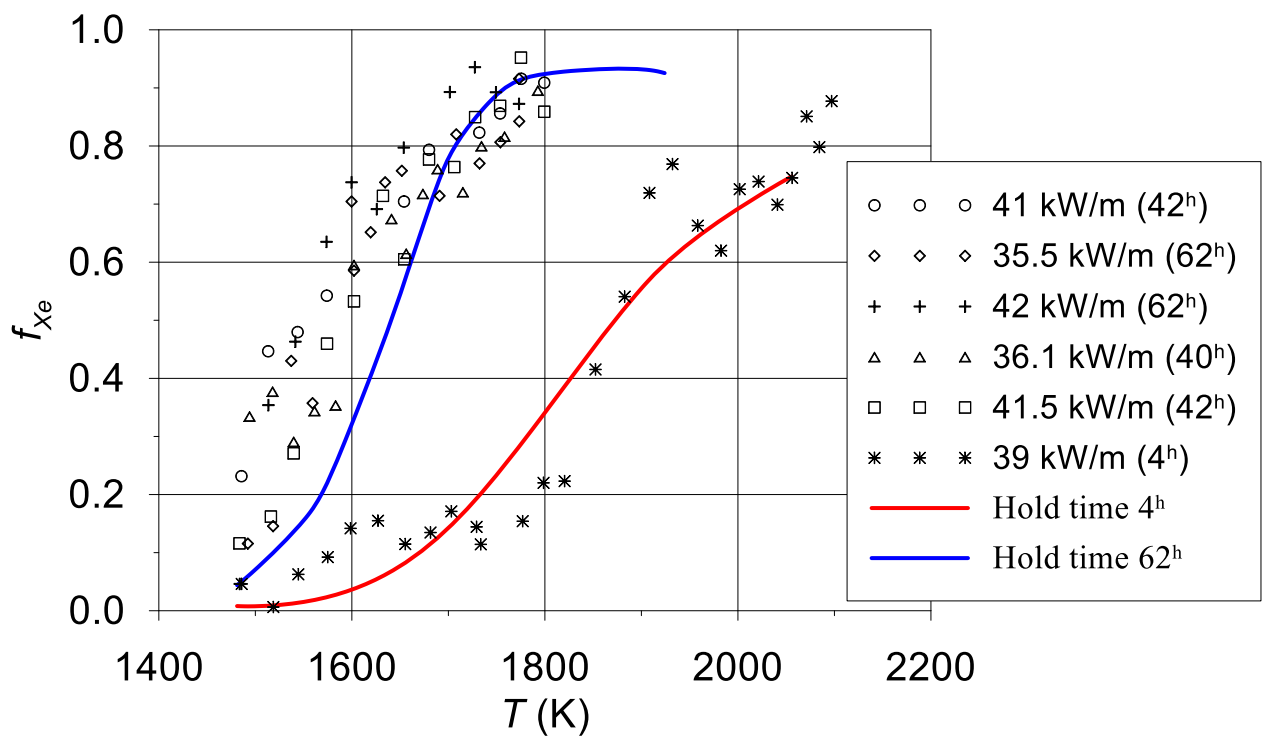

Fig. 4. Comparison of the measured (markers) and calculated (curves) radial distributions of the fractional gas release in the power ramp tests [6] with different hold times.

Consequently, calculation results for the gas release with the all above considered mechanisms switched on, are in a reasonable agreement with experimental results for different hold times (taking into account large uncertainties of $\approx 100-200 \mathrm{~K}$ (cf. [6]) in evaluation of local temperatures in the fuel pellet), as shown in Figure 4.

\section{Conclusions}

The main microscopic mechanisms of the intragranular bubble system evolution and fission gas release considered in the previous authors' paper [2] are briefly overviewed and further developed.

The modified Nelson model for gas atom resolution from bubbles under irradiation, taking into consideration a tendency of gas atoms ejected from a bubble into surrounding matrix to return back to this bubble by diffusion, results in significant microstructure changes characterised by the formation of a new population of large intragranular bubbles with a rather wide size distribution (from 30 to $500 \mathrm{~nm}$ ), observed in transient-tested fuel samples [5].

The MFPR calculations show that the main contribution to the gas release from fuel under transient conditions is associated with the bubble biased migration, whereas the random diffusion mechanism is relatively weak effect. Besides, the sweeping of bubbles by moving grain boundaries of growing grains also becomes an important mechanism of the gas release at high temperatures attained in the central zone of transient tested pellets. Superposition of all these mechanisms results in a reasonable prediction of the measured fractional gas release in the Ris $\varnothing$ ramp tests [6] with three different hold times of 3, 40 and $62 \mathrm{~h}$ at the terminal linear power of $\approx 40 \mathrm{~kW} / \mathrm{m}$, with except of the innermost zone of the cylindrical pellet, $x<0.1$, where the thermal gradient becomes small and the gas release is significantly underestimated.
In order to overcome this shortcoming of the previous calculations [2], the model was further improved by consideration of the additional mechanisms of bubbles transport to grain boundaries, considered earlier in MFPR in application to post-irradiation annealing regimes [7]. The model simulates time and spatial variation of the vacancy concentration in the presence of extended vacancy sources (grain boundaries and dislocations) and sinks (growing and coalescing intragranular bubbles), and being combined with the models for dislocation creep and for bubbles biased migration in the vacancy gradient, allows reasonable predictions of the gas release also in the central part of the fuel pellet, demonstrating self-consistency of the code calculations for the gas release and microstructure evolution.

\section{References}

1. I.L.F. Ray, H. Thiele, Hj. Matzke, J. Nucl. Mater. 188, 90 (1992)

2. M.S. Veshchunov, V.I. Tarasov, J. Nucl. Mater. 437, 250 (2013)

3. M.S. Veshchunov, V.D. Ozrin, V.E. Shestak, V.I. Tarasov, R. Dubourg, G. Nicaise, Nucl. Eng. Des. 236, 179 (2006)

4. M.S. Veshchunov, R. Dubourg, V.D. Ozrin, V.E. Shestak, V.I. Tarasov, J. Nucl. Mater. 362, 327 (2007)

5. M.S. Veshchunov, A.V. Boldyrev, V.D. Ozrin, V.E. Shestak, V.I. Tarasov, Nucl. Eng. Des. 241, 2822 (2011)

6. C. Bagger, M. Mogensen, C.T. Walker, J. Nucl. Mater. 211, $11(1994)$

7. M.S. Veshchunov, V.E. Shestak, Modelling of fission gas release from irradiated $\mathrm{UO}_{2}$ fuel under high temperature annealing conditions, J. Nucl. Mater. 430, 82 (2012)

8. P.G. Shewmon, Trans. AIME 230, 1134 (1964)

9. R.S. Nelson, J. Nucl. Mater. 31, 153 (1969)

10. M.S. Veshchunov, V.E. Shestak, J. Nucl. Mater. 376, 174 (2008) 
11. M.S. Veshchunov, A.V. Berdyshev, V.I. Tarasov, Development of fission gas bubble models for $\mathrm{UO}_{2}$ fuel in framework of MFPR code, in Preprint IBRAE-2000-08, Moscow (2000)

12. J.A. Turnbull, R.M. Cornell, J. Nucl. Mater. 41, 156 (1971)
13. J.A. Turnbull, R.M. Cornell, J. Nucl. Mater. 37, 355 (1970)

14. M.S. Veshchunov, J. Nucl. Mater. 346, 208 (2005)

15. M.S. Veshchunov, Materials 2, 1252 (2009)

16. M.S. Veshchunov, J. Nucl. Mater. 277, 67 (2000)

Cite this article as: Michael S. Veshchunov, Vladimir I. Tarasov, Development of the MFPR model for fission gas release in irradiated $\mathrm{UO}_{2}$ under transient conditions, EPJ Nuclear Sci. Technol. 3, 4 (2017) 